Submitted to and Accepted by

Journal of Applied Physics

\title{
Compression and strong rarefaction in high power impulse magnetron sputtering discharges
}

\author{
David Horwat ${ }^{1}$, André Anders ${ }^{2 *}$ \\ ${ }^{1}$ Institut Jean Lamour, Département CP2S, UMR 7198 CNRS-Nancy-Université-UPV-Metz, \\ Ecole des Mines de Nancy, Parc de Saurupt, CS14234, 54042 Nancy, France \\ ${ }^{2}$ Lawrence Berkeley National Laboratory, University of California, 1 Cyclotron Road, Berkeley, \\ California 94720, USA
}

November 11, 2010

\begin{abstract}
ACKNOWLEDGMENT
We thank the Inertial Fusion Group of Berkeley Lab for letting us use the multi-channel data acquisition system. We gratefully acknowledge the "France Berkeley Fund" for financial support of this collaboration. AA acknowledges support by the Erasmus Mundus Scholarship of the European Commission and by the Assistant Secretary for Energy Efficiency and Renewable Energy, Office of Building Technology, of the U.S. Department of Energy under Contract No. DE-AC02-05CH11231.
\end{abstract}

\section{DISCLAIMER}

This document was prepared as an account of work sponsored by the United States Government. While this document is believed to contain correct information, neither the United States Government nor any agency thereof, nor The Regents of the University of California, nor any of their employees, makes any warranty, express or implied, or assumes any legal responsibility for the accuracy, completeness, or usefulness of any information, apparatus, product, or process disclosed, or represents that its use would not infringe privately owned rights. Reference herein to any specific commercial product, process, or service by its trade name, trademark, manufacturer, or otherwise, does not necessarily constitute or imply its endorsement, recommendation, or favoring by the United States Government or any agency thereof, or The Regents of the University of California. The views and opinions of authors expressed herein do not necessarily state or reflect those of the United States Government or any agency thereof or The Regents of the University of California.

\footnotetext{
*Author to whom correspondence should be addressed. Electronic mail: aanders@lbl.gov.
} 


\title{
Compression and strong rarefaction in high power impulse magnetron sputtering discharges
}

\author{
David Horwat ${ }^{1}$, André Anders ${ }^{2 *}$ \\ ${ }^{1}$ Institut Jean Lamour, Département CP2S, UMR 7198 CNRS-Nancy-Université-UPV-Metz, \\ Ecole des Mines de Nancy, Parc de Saurupt, CS14234, 54042 Nancy, France \\ ${ }^{2}$ Lawrence Berkeley National Laboratory, University of California, 1 Cyclotron Road, Berkeley, \\ California 94720, USA
}

\begin{abstract}
Gas compression and strong rarefaction have been observed for high power impulse magnetron sputtering (HIPIMS) discharges using a copper target in argon. Time-resolved ion saturation currents of 35 probes were simultaneously recorded for HIPIMS discharges operating far above the self-sputtering runaway threshold. The argon background pressure was a parameter for the evaluation of the spatial and temporal development of the plasma density distribution. The data can be interpreted by a massive onset of the sputtering flux (sputter wind) that causes a transient densification of the gas, followed by rarefaction and the replacement of gas plasma by the metal plasma of sustained self-sputtering. The plasma density pulse follows closely the power pulse at low pressure. At high pressure, the relatively remote probes recorded a density peak only after the discharge pulse, indicative for slow, diffusive ion transport.
\end{abstract}

PACS: 52.80.Sm, 52.50.Dg, 52.40.Hf, 52.80.Tn, 81.15.Cd

\section{INTRODUCTION}

High Power Impulse Magnetron Sputtering (HIPIMS) is a fast developing plasma-based coatings technique. ${ }^{1-3}$ The main practical interest is in providing not just a flux of sputtered atoms but a flux of ions of the target material for energetic condensation, while keeping the cleanliness, controllability, and scalability of well-established magnetron sputtering processes. By "cleanliness" we primarily refer to the absence of macroparticles as they are typical for (unfiltered) cathodic arc deposition.

The physics of HIPIMS is generally understood due to the progress over the last few years (e.g. ${ }^{3-7}$ ). The presence of ionized sputtered atoms necessarily implies self-sputtering, which in some cases can dominate and sustain the discharge ${ }^{8-9}$. It has been shown that for some materials, a pulsed HIPIMS discharge can be sustained without gas, i.e. in high vacuum, by using the assistance of a short arc pulse to trigger the magnetron discharge. ${ }^{10-11}$ Eliminating the background gas and pushing the discharge far beyond the threshold of sustained self-sputtering produced ion currents at a substrate exceeding the discharge current. ${ }^{11}$ The condition for sustained self-sputtering can be written as $\Pi \equiv \alpha \beta \gamma_{S S}=1$, where $\alpha$ is the ionization probability, $\beta$ is the probability that the newly formed ion returns the target, and $\gamma_{S S}$ is the self-sputtering

\footnotetext{
* Author to whom correspondence should be addressed. Electronic mail: aanders@lbl.gov.
} 
yield. Before a sustained self-sputtering regime is established, a runaway phase with $\Pi>1$ exists, characterized by a rapid increase of the discharge current. ${ }^{11-13}$

Even as operation in vacuum is possible and interesting, magnetron sputtering is generally more stable when background gas is used to initiate and operate the discharge. Thus, the gas case remains of great practical importance and is therefore investigated in this study.

The average degree of ionization and ion charge state of the metal ions have been observed to increase with distance from the target for copper HIPIMS discharges in argon. ${ }^{14}$ It is thus relevant to consider the target-substrate distance as an important factor influencing the properties of produced films. For instance, a recent study considered the influence of this distance on the local chromium and titanium ion energy distribution functions. ${ }^{15}$ The study presented here will consider the plasma parameters at many locations relative to the target.

The flux of sputtered atoms during the HIPIMS pulse on-time is very high. Most of the sputtered atoms are slowed down by collisions with the background gas, especially if the background gas pressure is relatively high. ${ }^{16}$ The sputtered metal atoms become part of the process gas. This phenomenon includes gas heating and expansion, which is well known as rarefaction, initially described by Steenbeck for pulsed glow discharges ${ }^{17}$ and later by Rossnagel for DC magnetron discharges. ${ }^{18}$ In this paper we will look at the dynamics of the process for HIPIMS conditions, especially the compression that precedes rarefaction.

Compression and rarefaction can be expected to generate disturbances that propagate away from the target. Related phenomena have been found before. For example, for tantalum HIPIMS discharges of relatively short duration (70-100 $\mu \mathrm{s})$, electrostatic probe measurements showed that an ion-acoustic solitary wave with a plasma density of about $10^{19} \mathrm{~m}^{-3}$ can be generated. ${ }^{19-20}$ The wave traveled first away from the target. A second peak in the ion saturation probe current several $100 \mu \mathrm{s}$ after the end of the voltage pulse was observed, the timing of which depended on the dimensions of the chamber, which was taken as evidence for the reflection of the wave by the chamber walls.

The present study reports on investigations of the plasma making use of modern multichannel recording equipment that allows the ion density to be simultaneously measured at many places, thereby eliminating possible issues associated with drifts of discharge parameters, e.g. those caused by the unavoidable, progressing erosion of the target.

\section{EXPERIMENTAL SETUP}

The experiments were carried out using a $5 \mathrm{~cm}$ planar, unbalanced magnetron placed in a $1 \mathrm{~m}$ diameter and $200 \mathrm{~L}$ vacuum chamber as shown in Fig. 1 . The target was a $5 \mathrm{~mm}$ thick oxygen-free copper disc. The diameter of the circular racetrack was $25 \mathrm{~mm}$. The HIPIMS discharge was fed by an upgraded SPIK2000A pulse power supply (Melec $\mathrm{GmbH}$ ), capable of 500 A pulses, charged via a Pinnacle DC-supply (Advanced Energy). The onset of HIPIMS pulses of $1000 \mathrm{~V}$ and $400 \mu \mathrm{s}$ were assisted by very short ( $20 \mu \mathrm{s}), 200 \mathrm{~A}$, vacuum-arc discharges; this allowed us to extend the research from the traditional gas pressure (of order $1 \mathrm{~Pa}$ ) all the way down to the gasless regime, as previously reported. ${ }^{10-11}$ Most of this report will focus on the result with gas, while the results for the gasless case deserve a dedicated report. ${ }^{21}$ The influence of the trigger pulse is irrelevant at the relatively high pressures because the system triggers readily without the arc. At low pressure $(<1 \mathrm{~Pa})$, the arc plasma helps to avoid the common delay of discharge current known as the formative time lag. ${ }^{22}$ 
The pulse repetition rate was very low, only 10 pulses per second, to allow for high power pulses of relatively long duration while protecting the magnetron by observing its average power limit.

Pure argon gas was supplied near the target, establishing operational pressures of 0.13 , $0.4,0.9,1.75$ and $4.0 \mathrm{~Pa}$ adjusted by the specific combination of gas flow rate, up to $100 \mathrm{sccm}$ through a mass flow controller (MKS), and pumping speed via a cryogenic pump (Helix CryoTorr $^{\circledR}$ ) with finely adjustable gate valve (VAT). At fully opened valve, the pumping speed was $1500 \mathrm{l} / \mathrm{s}$ for air, and the chamber base pressure was at or below $10^{-4} \mathrm{~Pa}$, as measured by an ion gauge. The process pressure was monitored by a capacitance manometer. The discharge current was inductively recorded using a current transformer (Pearson ${ }^{\mathrm{TM}}$ model $301 \mathrm{X}, 0.01 \mathrm{VA}^{-1}, 2$ $\mathrm{MHz}$ bandwidth). The magnetic induction at the center of the target surface was $65 \mathrm{mT}$ (Gauss meter by F.W. Bell).

An array of 36 electrical probes consisting of $6 \mathrm{~mm}$ diameter stainless steel discs were positioned on an insulating plate at distances of $6,14,22$ and $30 \mathrm{~cm}$ from the center of the target surface and arranged along quarter circles (see Fig. 1). For each distance from the target center, one probe collects the current near the magnetron axis, and the others are off-axis. The angle between two consecutive trajectories between the probe and the target center is $10^{\circ}$. The probe holding plate was positioned parallel to the target axis and $1.5 \mathrm{~cm}$ below the axis to avoid a shadowing effect between two probes placed at two consecutive distances (for instance, a probe at $6 \mathrm{~cm}$ could shadow the probe at $14 \mathrm{~cm}$ ). Each probe was biased to $-100 \mathrm{~V}$ with respect to ground (anode potential) to record the time-dependent ion saturation current during a single pulse. The ion current was recorded using the voltage drop at a resistor selected such to ensure good signal to noise ratio. The data for all probes were simultaneously recorded with and stored in a 40-channel digital storage oscilloscope (National Instruments PXI-1045 chassis equipped with five PXI-5105 8-channel digitizer cards, with a maximum sampling rate of $60 \mathrm{MS} / \mathrm{second}$ per channel, operated under NI LabVIEW virtual instrument software). That is, the discharge current, applied target voltage pulse, and 36 time-dependent ion saturation currents were simultaneously recorded in the range $0-80^{\circ}$ relative to the target axis during a single pulse. The pulsed target voltage of $1000 \mathrm{~V}$ is not further displayed or discussed because it is constant for the $400 \mu$ s due to the large capacitance of the SPIK2000A unit. Unfortunately, the feedthrough for the probe at $22 \mathrm{~cm}, 40^{\circ}$ developed an irreparable short, thus its data were discarded and only 35 probe currents are reported.

\section{RESULTS}

The time evolution of the discharge current is displayed in Figure 2 for the initial argon pressures of $0.13,1.75$ and $4.0 \mathrm{~Pa}$. After the $1000 \mathrm{~V}$ voltage pulse has been shut off at $t=400$ $\mu \mathrm{s}$, the discharge current rapidly falls to zero and remains zero until the initiation of the next pulse. The magnitude of the initial peak and the following decay is more pronounced when the background gas pressure is high. The peak is small at the relatively low argon gas pressure of $0.13 \mathrm{~Pa}$ (and non-existent for the gasless case, not shown here). After the initial peak, the discharge current stabilizes at about 33 A regardless of the background gas pressure.

A survey of all time-dependent ion saturation currents at each electrostatic probe is displayed in Fig. 3 for the argon background pressures of $0.13,1.75$ and $4.0 \mathrm{~Pa}$. For best visibility and ease of interpretation, the time axis was kept the same for all conditions, while the axes of the ion saturation current were adjusted to show the signal very well. The absolute local ion density, which is approximately proportional to the measured ion current, decreases 
significantly as the distance from the target increases. The probes positioned in front of the target recorded a strong initial peak, whereas the probes under large angles showed a much weaker peak, or no peak at all. At large distances, the shapes of the ion density pulses strongly depend on the pressure even as the pressure is only modestly changed. After the end of the discharge pulse, the plasma decays rapidly close to the target, especially at low pressure, while the plasma "lingers" for a rather long time (milliseconds) at large distances.

Fig. 4 shows the case of $6 \mathrm{~cm}$ probes at a pressure of $1.75 \mathrm{~Pa}$ with greater resolution, and the inset has even higher time resolution to make clear that there is a fast and reproducible, partial drop at the end of the pulse but only for the probes positioned at $0^{\circ}, 10^{\circ}$ and $20^{\circ}$ from the discharge axis. The end of the discharge pulse at $400 \mu \mathrm{s}$ is indicated by the short, vertical arrows. Looking at the beginning of the pulses for the different angles, one clearly sees that the greatest initial peak is not at the center but appears under some angle of about $30^{\circ}$ to the target normal.

The ion current to probes at later times, much after the initial peak, appears to be independent of the gas pressure but depends on the location of the probe. Picking as an example the distribution at $6 \mathrm{~cm}$, measured for $0.13 \mathrm{~Pa}$, we obtain a cosine-type angular distribution (Fig. $5)$.

\section{DISCUSSION}

As mentioned in the introduction, gas rarefaction, i.e., the reduction of gas density in front of the magnetron target, is well known and investigated for DC magnetron sputtering ${ }^{18,23-}$ 26. Rarefaction is not uniform but depends on the process gas and the flux conditions from the target, where the flux includes sputtered atoms and to much lesser degree backscattered gas atoms (formerly primary ions hitting the target). Target materials of higher sputter yield have greater effects, and of course the mass ratio of sputtered atoms and gas atoms has a great influence on how energy and momentum is transferred from the sputter flux to the gas.

In DC sputtering, the transient at the beginning of the process is irrelevant for film deposition and was therefore not investigated. Considering steady-state conditions, Jimenez and coworkers ${ }^{26}$ concluded that both gas heating as well as the "sputter wind" should be included. In a simplified picture, rarefaction by gas heating can be understood looking at the ideal gas equation, where $n_{a}=p_{a} / k T_{a}$ indicates that the density is inversely reduced as the gas is heated; here $p_{a}$ is the gas pressure, which can be considered constant given a chamber that is typically much larger in volume than the heated volume of gas in front of the target. Rarefaction by "sputter wind", a term introduced by Hoffman, ${ }^{27}$ is related to momentum transfer and displacement of the process gas by the atoms coming from the target. In contrast to heating, the sputter wind has direction, which points away from the target.

Steenbeck investigated the ignition of glow discharges in the late 1920s and found that there is a decrease in density of the gas due to heating ${ }^{17}$ - perhaps the first report on rarefaction actually pointed out its dynamics! In HIPIMS, with its much stronger fluxes from the target during the pulse on-time compared to DC operation, rarefaction should be highly dynamic. Indeed, this has been mentioned several times ${ }^{12-13,28}$ and was simulated by Kadlec using a Direct Simulation Monte Carlo (DSMC) method. ${ }^{29}$

The peak observed at the beginning of the discharge current pulse (Fig. 2) is a direct signature of the pressure dynamics in the vicinity of the target. As ionization of the background gas becomes strong, the sputtered atoms from the target collide with the gas atoms and the onset of "sputter wind" leads to a compression of the gas, thereby providing transient conditions for 
the generation of an even stronger ion current to the target. The massive onset of sputtering acts like a piston on the process gas, and its compression will depend on both the initial density as well as the gradient with which the sputter flux starts.

What happen next depends much on the specific conditions. If ionization of the sputtered atoms remains modest, self-sputtering cannot sustain itself and the onset of rarefaction will greatly reduce the ion current to the target, as shown for sub-runaway conditions (c.f. curve I(530 $\mathrm{V})$ in Fig. 1 of ref. $^{13}$ ). Should self-sputtering run away, i.e., $\Pi>1$, the current to the target will greatly increase but then decrease - due to rarefaction - before settling on a steady-state value. Given that we work with copper and apply $1000 \mathrm{~V}$ pulses, we can be sure that we always operate in the latter regime in this study: ${ }^{1-13}$ all curves in Figs. 2-4 refer to this situation. After steady state is established ( $\Pi=1$ for $t>100 \mu \mathrm{s})$ we found that the discharge current (Fig. 2) and the ion probe currents near the target $(6 \mathrm{~cm}$, top row of Fig. 3$)$ are practically independent of the initial gas pressure, which is a convincing argument for very strong rarefaction.

The measurements at the various locations allow us to recognize further details. The sputter wind is mainly directed normal from the target, although some work indicated that once atoms of the sputter wind are ionized, they may return to the target if produced close to it, or escape to the substrate and nearby regions. Their motion is affected by the potential distribution of the magnetic presheath ${ }^{7}$ and may also be affected by the magnetized electrons such that they tend to move off axis, ${ }^{5,14}$ which may be related to the anomalous transport of electrons across magnetic field lines. The transport is called "anomalous" because it has been shown to exceed the Bohm current. ${ }^{30}$ We see that the ion density peak measured by probes close to the target ( 6 $\mathrm{cm}$ ) is higher at about $20^{\circ}-30^{\circ}$ than the ion density peak on axis (Figs. 3 top row and 4 ).

Another, perhaps more straight forward effect and explanation is to consider rarefaction of the process gas before the HIPIMS pulse has started! Such effect has been seen with hot niobium targets, ${ }^{7}$ where the density of the pre-target region is reduced due to the high temperature of the target caused by previous pulses. Working with the very same magnetron here, and using the same setup, it is reasonable to also consider elevated target temperature. This would reduce the local gas density near the target center, and thus lead there to less compression and rarefaction.

At larger distances, though, the magnetron looks increasingly like a point source and the ion density maximum can be found on axis.

The strength of the compression and responding rarefaction can be derived from the sharpness of the initial peak as measured by the amplitude and full width at half maximum (FWHM). Looking at the probes closest to the target, at $6 \mathrm{~cm}$, top row of Fig. 3, we see that not much compression can occur at the lowest pressure of $0.13 \mathrm{~Pa}$, while sharp peaks are seen at higher pressure. The FWHM at $4 \mathrm{~Pa}$ is greater than at $1.75 \mathrm{~Pa}$, likely because the smaller mean free path and greater collision frequency at $4 \mathrm{~Pa}$ may already have dissipated some of the compression wave before it arrived at those probes. This argument is supported by looking at the next row, the probes at $14 \mathrm{~cm}$. Here, the initial peak at $1.75 \mathrm{~Pa}$ is broader than at $6 \mathrm{~cm}$, and practically merged with the self-sputtering signal. At even greater distances, no initial peaks can be identified.

Another nice illustration of compression and rarefaction is the case of Fig. 3, $1.75 \mathrm{~Pa}, 14$ $\mathrm{cm}$. Probes at angles up to $60^{\circ}$ show an initial peak, which shows reduced amplitude as we go to greater angles, and probes at $70^{\circ}$ and $80^{\circ}$ show no such peak at all. Those observations are consistent with the sputtering wind going mostly forward. 
While the ion current curves, corresponding to local ion density, can tell a lot about the dynamics of the process, the information on the ion transport mechanism is limited. We see that probes at greater distance record vastly different signals depending on the background gas pressure, indicating the importance of collisions at pressures of about $1 \mathrm{~Pa}$ or greater. As long as the gas density is low, even the remote probes can register signals that resemble the discharge power pulse shape, though with some delay associated with ion transport to the probe. Using a time-of-flight approach allowed us to derive energy distribution functions for the case of gasless operation (reported elsewhere ${ }^{21}$ ). For operation in gas, however, the concept of time of flight measurements must be questioned or even abandoned due to the collisions, and hence no velocity or kinetic energy data will be reported here. However, we can see that the maximum ion densities at large distances (e.g. $22 \mathrm{~cm}$ or $30 \mathrm{~cm}$ ) at high pressure are recorded after the discharge pulse. The long time between the discharge end and the observed ion peak indicates slow transport in which collisions play an important role, e.g. ambipolar diffusion with anomalous electron transport. ${ }^{30}$

The ion current pulses for the $0^{\circ}, 10^{\circ}$ and $20^{\circ}$ probes exhibited a reproducible fast drop at the end (Fig. 4) which cannot be explained by electromagnetic noise; rather, it can be related to backscattered particles. A strong argument is that the effect can only be observed for the probes in front of the target at the relatively small distance of $6 \mathrm{~cm}$ (Fig. 3, top row and Fig. 4 with higher resolution). The amplitude of the drop, which is typically $5 \%$ of the total ion current, also speaks in favor of this interpretation. This observation was unexpected and opens the door to more careful measurements in the future with higher resolution: possibly a simple, low cost approach to distinguish between energetic ions that originate from backscattered particles and lower energy particles originating from sputtering.

Finally, looking at Fig. 5, we find that the ion currents measured at $6 \mathrm{~cm}$ from the target center for different angles to the target normal exhibit a cosine-type distribution. The best fit for the experimental data is $j_{i}(\theta)=C \cos ^{3 / 2}(\theta)$, where $\mathrm{C}$ is a constant and $\theta$ is the angle to the target normal. This empirical relationship is in agreement with the usual angular dependencies for sputtered atoms found for a broad range of materials and conditions. ${ }^{31}$ It indicates that at the low background pressure of $0.13 \mathrm{~Pa}$, only few collisions have happened such that the original distribution is approximately preserved. The slightly flattened top of the angular distribution, near $\theta=0$, can be related to the finite target size (not a point source) and to the already discussed rarefaction before the pulse due to elevated target temperature. Fig. 5 corresponds to the data shown in Fig. 3, left of the upper row. Going to higher pressures, i.e. to the right in this row, we see that the angular distribution is almost the same considering later times, $t>100 \mu \mathrm{s}$, which again is due to the replacement of argon by copper and the establishment of sustained selfsputtering. Many of the here-reported results call for detailed modeling, and they can serve to validate future models.

\section{CONCLUSIONS}

The plasma density distribution for long $(400 \mu \mathrm{s})$ HIPIMS pulses was investigated using 35 probes at various locations relative to the target. The local ion currents, representing the local plasma density, showed numerous features which depend on the location and initial gas pressure. All of the features can be explained in terms of gas compression followed by rarefaction and replacement of the gas by copper plasmas operating in the sustained self-sputtering mode.

At the relatively low pressure of $0.13 \mathrm{~Pa}$, the influence of the gas is weak and the ion density pulse shapes closely follow the shape of the power pulse. Probes close to the target (6 
and $14 \mathrm{~cm}$ ) register a small initial peak associated with compression and rarefaction. At $22 \mathrm{~cm}$, this peak is primarily seen close to the normal direction, which is associated with the directionality of the "sputter wind." At the even larger distance of $30 \mathrm{~cm}$, the initial compression peak is largely dissipated and thus very small.

At higher pressure, e.g. $1.75 \mathrm{~Pa}$, the compression-rarefaction peaks are very pronounced. At even higher pressure, $4 \mathrm{~Pa}$, the compression peak is broadened by collisions and merges with the plasma peak generated by self-sputtering. For all pressures, the ion density strongly decreases with distance from the magnetron. The shape of the ion density pulse carries the signature of collisions: at high pressure, the maximum density is observed only after the termination of the discharge pulse, indicating relatively slow transport.

\section{ACKNOWLEDGMENTS}

We thank the Inertial Fusion Group of Berkeley Lab for letting us use the multi-channel data acquisition system. We gratefully acknowledge the "France Berkeley Fund" for financial support of this collaboration. AA acknowledges support by the Erasmus Mundus Scholarship of the European Commission and by the Assistant Secretary for Energy Efficiency and Renewable Energy, Office of Building Technology, of the U.S. Department of Energy under Contract No. DE-AC02-05CH11231. 


\section{References}

1 U. Helmersson, M. Lattemann, J. Bohlmark, A. P. Ehiasarian, and J. T. Gudmundsson, Thin Solid Films 513, 1 (2006).

2 A. Ehiasarian, in Plasma Surface Engineering Research and its Practical Applications, edited by R. Wei (Research Signpost, Kerala, India, 2008), pp. 35-86.

3 K. Sarakinos, J. Alami, and S. Konstantinidis, Surf. Coat. Technol. 204, 1661 (2010).

$4 \quad$ J. Vlček, P. Kudláček, K. Burcalová, and J. Musil, Eur. Phys. Lett. 77, 45002 (2007).

5 D. Lundin, P. Larsson, E. Wallin, M. Lattemann, N. Brenning, and U. Helmersson, Plasma Sources Sci. Technol. 17, 035021 (2008).

6 M. Hala, N. Viau, O. Zabeida, J. E. Klemberg-Sapieha, and L. Martinu, J. Appl. Phys. 107, 043305 (2010).

7 A. Anders, Surf. Coat. Technol., submitted (2011).

$8 \quad$ W. M. Posadowski and Z. Radzimski, J. Vac. Sci. Technol. A 11, 2980 (1993).

9 A. Wiatrowski, W. M. Posadowski, and Z. J. Radzimski, J. Vacuum Sci. Technol. A 26, 1277 (2008).

10 J. Andersson and A. Anders, Appl. Phys. Lett. 92, 221503 (2008).

11 J. Andersson and A. Anders, Phys. Rev. Lett. 102, 045003 (2009).

12 A. Anders, J. Andersson, and A. Ehiasarian, J. Appl. Phys. 102, 113303 (2007).

13 A. Anders, J. Andersson, and A. Ehiasarian, J. Appl. Phys. 103, 039901 (2008).

14 D. Horwat and A. Anders, J. Phys. D: Appl. Phys. 41, 135210 (2008).

15 A. P. Ehiasarian, J. Andersson, and A. Anders, J. Phys D: Appl. Phys. 43, 275204 (2010).

16 W. D. Westwood, J. Vac. Sci. Technol. 15, 1 (1978).

17 M. Steenbeck, Wiss. Veröff. Siemens-Konzern 9, 42 (1930).

S. M. Rossnagel, J. Vac. Sci. Technol. A 6, 19 (1988).

J. Alami, J. T. Gudmundsson, J. Bohlmark, J. Birch, and U. Helmersson, Plasma Sources Sci. Technol. 14, 525 (2005).

K. B. Gylfason, J. Alami, U. Helmersson, and J. T. Gudmundsson, J. Phys. D: Appl. Phys. 38, 3417 (2005).

D. Horwat and A. Anders, Appl. Phys. Lett., accepted for publication (2010).

A. Kersch, W. Morokoff, and C. Werner, J. Appl. Phys. 75, 2278 (1994). 
G. M. Turner, J. Vac. Sci. Technol. A 13, 2161 (1995).

25 A. Palmero, H. Rudolph, and F. Habraken, Thin Solid Films 515, 631 (2006).

26 F. Jimenez, S. D. Ekpe, and S. K. Dew, J. Vacuum Sci. Technol. A 24, 1530 (2006).

27 W. D. Hoffman, J. Vac. Sci. Technol. A 3, 561 (1985).

D. Lundin, N. Brenning, D. Jädernäs, P. Larsson, E. Wallin, M. Lattemann, M. A. Raadu, and U. Helmersson, Plasma Sources Sci. Technol. 18, 045008 (2009).

S. Kadlec, Plasma Processes and Polymers 4, S419 (2007).

D. Lundin, U. Helmersson, S. Kirkpatrick, S. Rohde, and N. Brenning, Plasma Sources Sci. Technol. 17, 025007 (2008).

31 H. Gnaser, in Sputtering by Particle Bombardment, edited by R. Behrisch and W. Eckstein (Springer, Berlin, 2007), pp. 231-327.

\section{Figure Captions}

FIG. 1. Schematic view of the experimental setup.

FIG. 2. Time dependent discharge current for $0.13,1.75$ and $4 \mathrm{~Pa}$ argon background pressure; copper target, the applied voltage was $1000 \mathrm{~V}$, which was constant during the $400 \mu \mathrm{s}$ pulse.

FIG. 3. Time-dependent ion saturation currents for $0.13 \mathrm{~Pa}, 1.75 \mathrm{~Pa}$ and $4 \mathrm{~Pa}$ at $6,14,22$ and $30 \mathrm{~cm}$ from the target and for collection angles varying from 0 to $80^{\circ}$ to the normal of the target as indicated in Fig 1. Note that the timescale was kept constant for better comparison but the current amplitude is differently displayed for each distance.

FIG. 4 Data from the probes at $6 \mathrm{~cm}$ distance and $1.75 \mathrm{~Pa}$ argon background pressure shown with higher resolution. The small vertical arrows indicate the moment of termination of the applied voltage at $400 \mu \mathrm{s}$, and the inset shows a reproducible, rapid drop which occurs right after voltage pulse termination but only for those probes that are directly in front of the target surface $\left(0^{\circ}, 10^{\circ}\right.$ and $\left.20^{\circ}\right)$.

FIG. 5 Distribution of the ion saturation current, indicating copper ion density, as measured at $t=200 \mu$ s for various angles to the normal at a distance of $6 \mathrm{~cm}$ from the target center. The background pressure was $0.13 \mathrm{~Pa}$. 


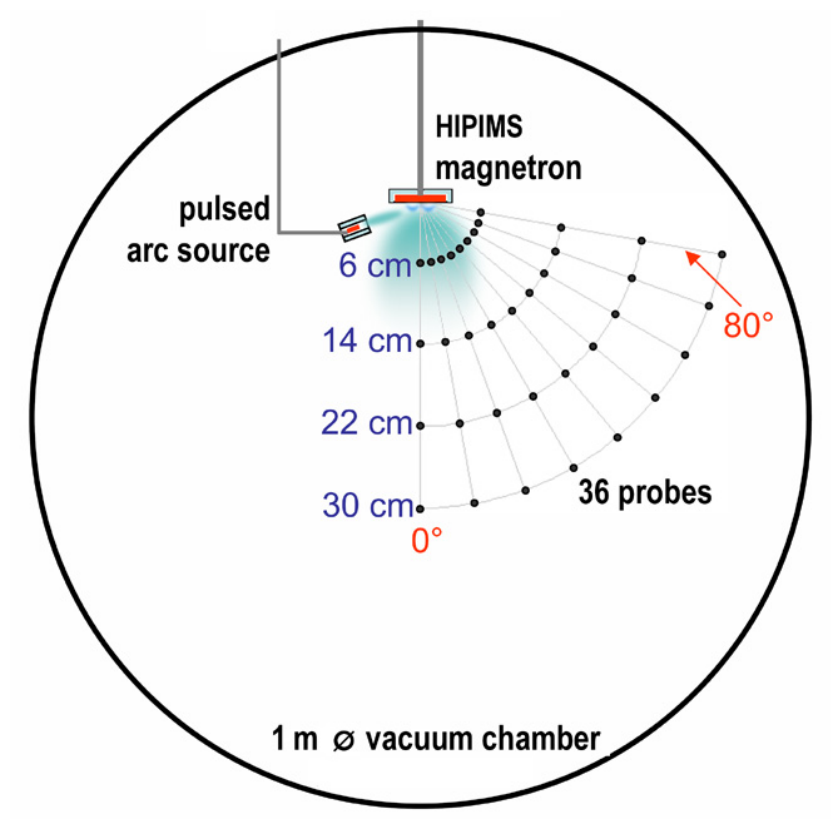

Fig. 1 


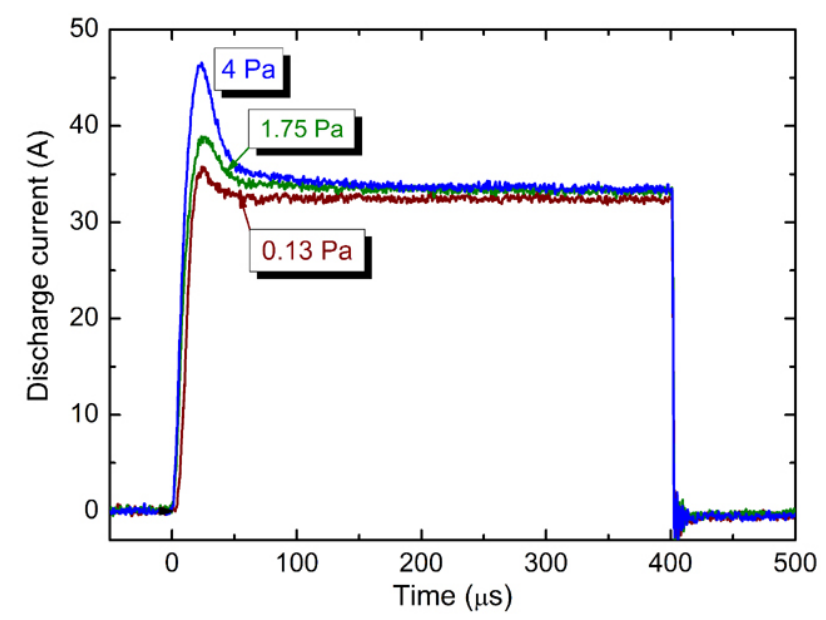

Fig. 2 

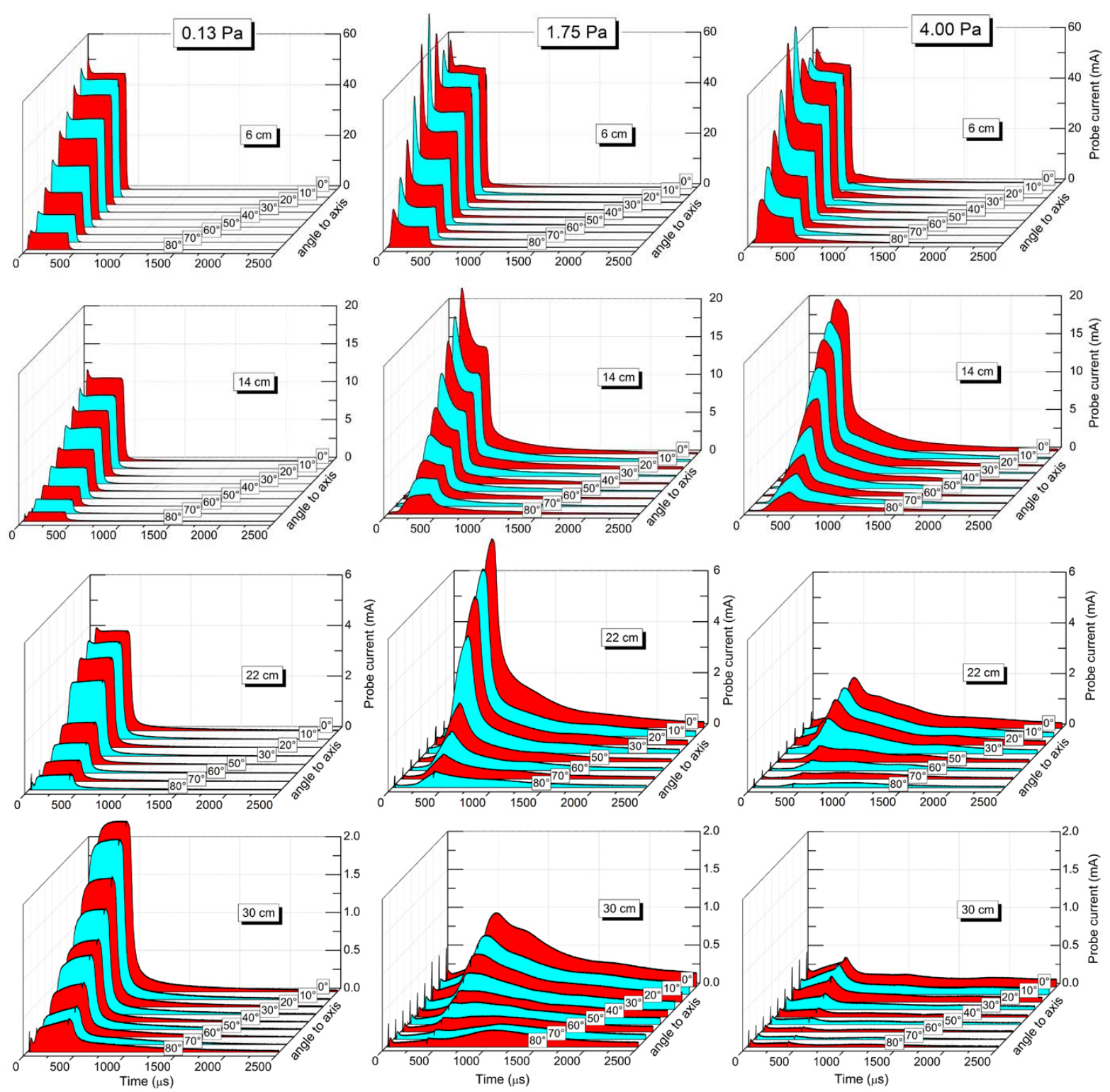

Fig. 3 


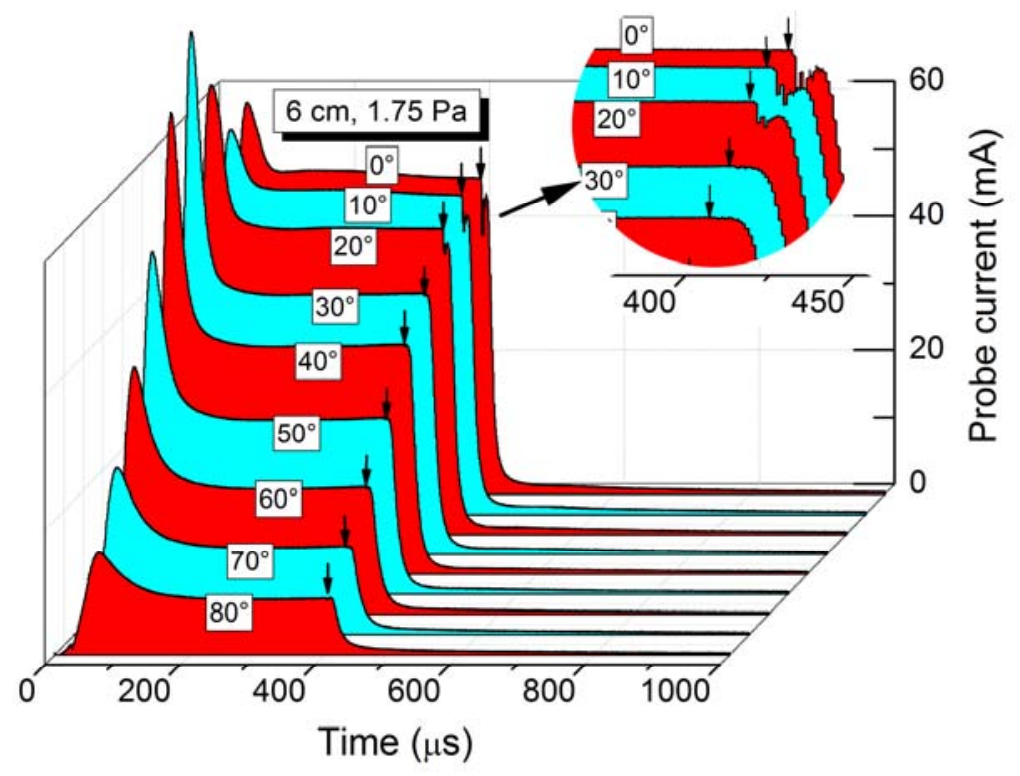

Fig. 4 


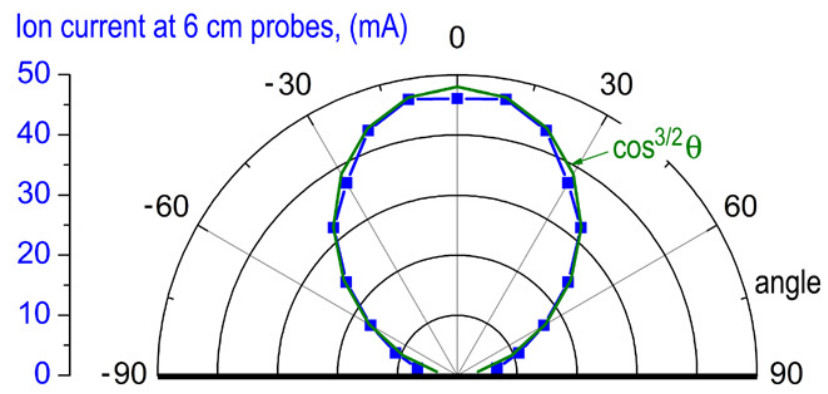

Fig. 5 